\title{
Survival difference associated with controlled-release oxycodone analgesic therapy for oxaliplatin-induced peripheral neuropathy in advanced colorectal cancer patients
}

\author{
Makoto Nagashima*, Ayako Hiranuma, Takashi Oshiro, Yu Sato, Tomoaki Kitahara, Taiki Nabekura, Yuki Moriyama, Motoaki Arai, \\ Mika Ando, Kengo Kadoya, Ayami Sato, Kentaro Kawamitsu, Ryuichi Takagi, Tasuku Urita, Yutaka Yoshida, Hiroshi Tanaka and Shinichi \\ Okazumi \\ Department of Surgery, Toho University Sakura Medical Center, Japan
}

\begin{abstract}
Oxaliplatin is a platinum-based cytotoxic chemotherapeutic agent, and is usually used in combination; as part of the FOLFOX regimen, to treat advanced colorectal cancer (CRC) patients. However, oxaliplatin produces significant chemotherapy-induced peripheral neuropathy (CIPN), a potential dose-limiting side effect of treatment. Preventive and therapeutic protocol for oxaliplatin-induced peripheral neuropathy (OIPN) has not yet been established. Previously, we reported that controlled-release oxycodone (CR oxycodone) attenuated the pain of OIPN and extended FOLFOX therapy in advanced CRC patients. We further investigated the efficacy of CR oxycodone for OIPN and its association with patients' survival time.
\end{abstract}

This was a retrospective analysis of advanced CRC patients. A total of 64 patients with stage III or IV CRC were included in this study. All patients underwent surgery to extirpate the primary CRC and received curative-intent FOLFOX chemotherapy. Patients who were administered CR oxycodone during the FOLFOX therapy period were defined as the OXY group (29 cases), and those who did not receive CR oxycodone treatment were defined as the non-OXY group (35 cases). Survival time was calculated using the Kaplan-Meier method to determine differences between patients in the OXY and the non-OXY groups.

Patients in the OXY group had relatively longer survival than those in the non-OXY group (median survival, 58 months vs. 36 months; $P=0.06$ ).

Early administration of CR oxycodone for OIPN might be relatively effective for better patient compliance with FOLFOX chemotherapy, a better QOL, and longer survival in patients with advanced CRC.

\section{Introduction}

The incidence of colorectal cancer (CRC) is relatively high in many developed countries. Cancer Statistics in Japan, 2016 reported that the colon/rectum is the third leading site for cancer occurrence in men and second in women. Nevertheless, deaths from CRC have decreased gradually over the past several years. This trend is observed in many developed countries. It is possible that development of new anti-cancer therapeutic options is one of the most important contributory factors in the decreased mortality rate and improved survival in CRC patients.

Oxaliplatin is a third-generation platinum-based cytotoxic chemotherapeutic agent. Oxaliplatin is commonly used to treat CRC that has spread or metastasized [1,2]. It is usually given in combination, with other anticancer agents (5-fluorouracil and leucovorin) as the FOLFOX chemotherapeutic protocol. Even though oxaliplatin is active against advanced CRC, it causes significant chemotherapy-induced peripheral neuropathy (CIPN), which is a potential dose-limiting side effect of this drug $[3,4]$.

Two spectrums of peripheral neuropathy are associated with oxaliplatin: acute neuropathy, which occurs immediately following infusion, and chromic neuropathy, which occurs with chronic administration. Significant acute neuropathy occurs in most patients
(85-95\%), developing during or immediately following infusion, and typically resolving within a week. The most common symptoms are distal limb and mouth paresthesia exacerbated by exposure to cold, jaw pain on biting, and oral numbness. Motor signs and symptoms may also occur, including muscular spasm-like contractions, fasciculations, and cramps [5].

Chronic oxaliplatin-induced peripheral neuropathy (OIPN) is dose-dependent, with severe neuropathy typically occurring in $10 \%$ to $20 \%$ of patients at a cumulative dose of $750 \mathrm{mg} / \mathrm{m}^{2}$ to $850 \mathrm{mg} / \mathrm{m}^{2}$. The incidence of neuropathy increased sharply with cumulative dose, with severe neuropathy estimated to occur in $10 \%$ of patients after 9 treatment cycles and in $50 \%$ after 14 treatment cycles [6].

*Correspondence to: Makoto Nagashima, Department of Surgery, Toho University Sakura Medical Center, 564-1 Shimoshizu, Sakura 285-8741, Japan, Tel: +81-43-462-8811, Fax: +81-43-463-1456, E-mail: nagashima@sakura.med. toho-u.ac.jp

Key words: oxycodone, oxaliplatin, chemotherapy induced peripheral neuropathy, colorectal cancer, FOLFOX

Received: September 12, 2018; Accepted: September 28, 2018; Published: October 01, 2018 
Nagashima M (2018) Survival difference associated with controlled-release oxycodone analgesic therapy for oxaliplatin-induced peripheral neuropathy in advanced colorectal cancer patients

OIPN is a highly distressing symptom and often interferes with the patient's daily activities with substantial decrease in quality of life (QOL) $[7,8]$. So far, preventive and therapeutic protocols have not established; therefore, dose reduction or discontinuations of the drug are the only options when symptoms become severe. The recent prevention and treatment guidelines for CIPN released by the American Society of Clinical Oncology (ASCO) concluded that there is no recommended preventive agent and duloxetine was recommended as a therapeutic option. In this ASCO guideline, opioids administered to the patients were excluded in the analysis due to lack of randomized control trials (RCTs). Although some case series suggested the efficacy of opioids for CIPN, opioid use in this condition is still controversial [9].

Even though the effect of chemotherapy on survival in CRC patients has been extensively studied, the correlation between therapeutic agents for OIPN and patient survival has not been examined. Previously, we reported that controlled-release oxycodone (CR oxycodone) was effective for painful OIPN and contributed to patients tolerating extended FOLFOX therapy in advanced CRC patients [10]. Based on these results, in this study, we have further evaluated the long-term efficacy of CR oxycodone for OIPN and its association with patient survival time.

\section{Material and methods}

\section{Patients}

We carried out a retrospective analysis of a cohort of patients with histologically confirmed advanced CRC between November 2006 and February 2012 at the Toho University Sakura Medical Center (Sakura, Japan). We reviewed the medical records, and included a total of 64 patients with stage III or IV disease in this study. All patients underwent surgery to extirpate the gross lesion of the primary CRC and subsequently received curative-intent oxaliplatin-based FOLFOX therapy. All patients received modified FOLFOX6 (mFOLFOX6) chemotherapy. Molecular targeted therapies (bevacizumab and panitumumab) were administered prior to mFOLFOX6 in 27 cases. Oxaliplatin-based FOLFOX chemotherapy was continued until disease progression (PD), decision to administer alternative therapies, development of unacceptable toxicities, or patient rejection of further treatment. The patients were reported in our previous study (Table 1) [10].

Table 1. Characteristics of the Oxy Group and the Non-OXY Group

\begin{tabular}{|l|c|c|}
\hline & OXY Group & Non-OXY Group \\
\hline Number & 29 & 35 \\
\hline Mean age (range) & $62.7(48-75)$ & $64.9(48-76)$ \\
\hline Gender & & \\
\hline Male & 16 & 20 \\
\hline Female & 13 & 15 \\
\hline Stage & & \\
\hline III & 4 & 12 \\
\hline IV & 25 & 23 \\
\hline Chemotherapy & & 28 \\
\hline mFOLFOX & 9 & 7 \\
\hline mFOLFOX+MTT & 20 & $(2-18)$ \\
\hline Median of FOLFOX cycles & $(6-46)$ & $483.0 \mathrm{mg} / \mathrm{m}^{2 *}$ \\
\hline (range) & $1072.3 \mathrm{mg} / \mathrm{m}^{2 *}$ & \\
\hline Median of cumulative oxaliplatin dose & $\left(408.7-3385.3 \mathrm{mg} / \mathrm{m}^{2}\right)$ & $\left(76.2-1414.1 \mathrm{mg} / \mathrm{m}^{2}\right)$ \\
\hline (range) & & \\
\hline "P $P$ 0.05 \\
MTT: Molecular Target Therapies & & \\
\hline
\end{tabular}

\section{Study design}

Patients were divided into two groups. Patients with cancer-related pain who had been administered CR oxycodone during the FOLFOX therapy period were defined as the OXY group (29 cases). The daily $\mathrm{CR}$ oxycodone doses at baseline ranged from 10 to $40 \mathrm{mg}$. No opioids besides $\mathrm{CR}$ oxycodone were administered to the study population before and during FOLFOX therapy. The maximum daily CR oxycodone doses were as follows: $160 \mathrm{mg}$ for one patient, $40 \mathrm{mg}$ for two patients, $30 \mathrm{mg}$ for three patients, $20 \mathrm{mg}$ for three patients, $15 \mathrm{mg}$ for two patients, and $10 \mathrm{mg}$ for 18 patients. Patients who did not receive CR oxycodone were defined as the non-OXY group (35 cases). Neurological toxicities were assessed according to the Common Terminology Criteria for Adverse Events (CTCAE) version 3.0 (U.S. Department of Health and Human Services, National Institutes of Health, National Cancer Institute). Survival time was calculated from the date of enrollment to the date of death. The study was designed to determine the differences in survival time between patients in the OXY and non-OXY groups, undergoing oxaliplatin-based FOLFOX therapy. Survival time was calculated using of the Kaplan-Meier method. Data from patients who were alive at the last follow-up (June 11, 2018) were censored.

\section{Statistical analysis}

For both treatment groups, the medians and relative frequencies were compared using Welch's $t$-test for two independent samples and the Wilcoxon signed-rank test and Fisher's exact test, respectively. Statistical significance was determined as $P<0.05$.

\section{Characteristics of the eligible patients}

All patients in this study had OIPN. Severe sensory neuropathy (grade 3) was not observed in the OXY group; however it was observed in the non-OXY group ( 2 cases). The median numbers of FOLFOX cycles in the OXY group (13, range 6-46) was more than that in the non-OXY group (7, range 2 -18) $(P<0.05$; Welch's $t$-test). The median cumulative oxaliplatin dose in the OXY group $\left(1072.3 \mathrm{mg} / \mathrm{m}^{2}\right.$, range $408.7-3385.3 \mathrm{mg} / \mathrm{m}^{2}$ ) was also more than that in the non-OXY group $\left(483.0 \mathrm{mg} / \mathrm{m}^{2}\right.$, range $\left.76.2-1414.1 \mathrm{mg} / \mathrm{m}^{2}\right)(P<0.05$; Welch's $t$-test) (Table 1). CR oxycodone might extend the use of FOLFOX therapy, and then, the patients in the OXY group had more opportunities to be treated by administration of Molecular targeted therapies.

\section{Results}

Patient survival time was relatively longer in the OXY group than in the non-OXY group (Figure 1). Median estimates of overall survival were as follows: 51 months (95\% confidence interval (CI), 32 to 70 ) in the entire samples (64 patients), 58 months ( $95 \%$ CI, 37.5 to 78.5$)$ in the OXY group (29 patients) and 36 months (95\% CI, 28.9 to 43.1 ) in the non-OXY group ( 35 patients) ( $P=0.06$ using the Wilcoxon signed-rank test). Hazard ratio (HR) for death in the OXY group was 0.68 (95\% CI, 0.38 to $1.22, P=0.19$ ).

\section{Discussion}

Opioids have been regarded to be effective for the treatment of several forms of neuropathic pain. RCTs have demonstrated the efficacy of CR-oxycodone in the treatment of diabetic neuropathy $[11,12]$. A systematic review of literature conducted by the American Academy of Neurology concluded that CR oxycodone was effective for postherpetic neuralgia (PHN) pain [13]. A postmarketing surveillance study demonstrated that CR oxycodone was effective in the management of moderate and severe PHN pain with a fast onset of pain relief 


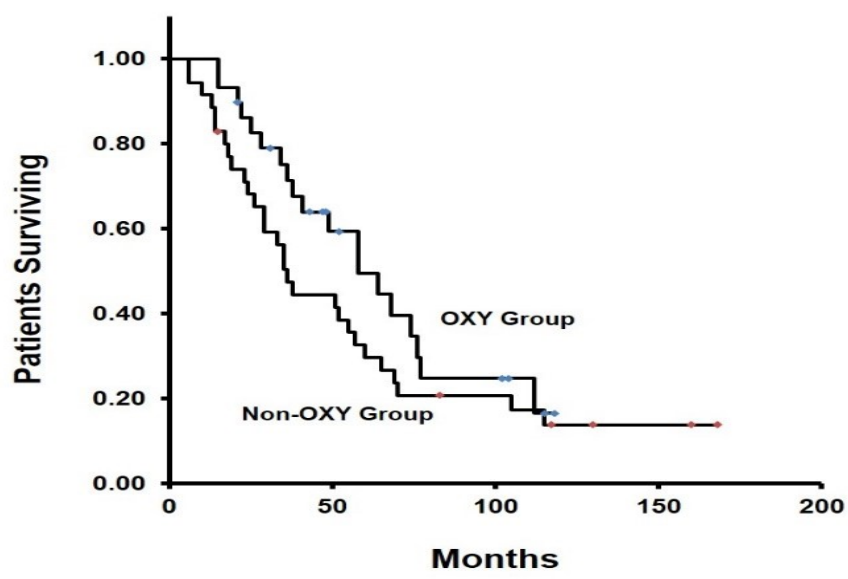

Figure 1. Overall survival for each study group estimated using the Kaplan-Meier method. Tick marks indicate censoring of data

after administration and it also improved the patients' sleep quality [14]. Oxycodone showed a profound antinociceptive effect under a neuropathic pain-like state in mice with less of a rewarding effect [15].

As per ASCO practice guidelines, no agents are recommended for the prevention of CIPN. With regard to the treatment of existing CIPN, the best available data supported a moderate recommendation for duloxetine [9]. Pachman et al. examined the existing evidence for both CIPN and non-CIPN treatments and developed a summary of the best available evidence with the aim of developing a practical approach for the treatment of CIPN, based on available literature and clinical practice experience. They reported that opioids had been shown to be effective for the treatment of a variety of forms of neuropathic pain. RCTs have demonstrated the efficacy of CR oxycodone in the treatment of diabetic neuropathy. Opioids were often recommended as second or third line treatments for non-CIPN neuropathic pain in various consensus statements. The study concluded that given the limited definitive data in CIPN, extrapolation of a treatment algorithm from the non-CIPN neuropathic pain literature might be rational for the treatment of CIPN [16].

Bortezomib, a dipeptide boronic acid inhibitor of the $26 \mathrm{~S}$ proteasome, is used in the treatment of multiple myeloma. Bortezomib induces chronic peripheral neuropathy. Cartoni et al. indicated that CR oxycodone was effective and well tolerated for relief of bortezomibinduced peripheral neuropathy and improved the patients' QOL during treatment [17].

Temel et al. indicated that among patients with metastatic non-small-cell lung cancer, early palliative care led to significant improvements in both QOL and mood. Patients receiving early palliative care required less aggressive care at the end of life but had longer survival, compared to patients receiving standard care [18]. Previous reports have shown that a lower QOL and depressed mood are associated with shorter survival among patients with metastatic non-small-cell lung cancer [19]. The integration of supportive care and early palliative care with standard chemotherapy might improve QOL, and facilitate the appropriate administration of anticancer agents. Better management of side effect produced by oncogenic care can lead to stable conditions and prolong survival in advanced cancer patients.

Previously, we retrospectively investigated the efficacy and tolerability of CR oxycodone for OIPN during FOLFOX therapy in CRC patients. Grade 3 severe sensory neuropathy was observed only in the
non-OXY group, not in the OXY group. All patients in the OXY group completed the scheduled FOLFOX therapy until PD, whereas $28.6 \%$ of patients in the non-OXY group terminated FOLFOX therapy due to severe peripheral neuropathy. The average number of FOLFOX cycles in the OXY group (13 cycles) significantly exceeded that in the nonOXY group ( 7 cycles). The cumulative dose of administered oxaliplatin was also significantly higher in the OXY group $\left(1072.3 \mathrm{mg} / \mathrm{m}^{2}\right)$ than in the non-OXY group $\left(483.0 \mathrm{mg} / \mathrm{m}^{2}\right)$ (Table 1) [10].

In the present study, we compared the overall survival between the OXY group and the non-OXY group. Median overall survival was 58 months and 36 months in the OXY group and the non-OXY group, respectively $(P=0.06)$ (Figure 1$)$. The CRC patients receiving CR oxycodone treatment had less OIPN, received more FOLFOX chemotherapy, and had relatively longer survival.

Chronic CIPN remains after conclusion of chemotherapy. 30\% of patients who received platinum-based chemotherapy continue to experience discomfort and diminish their QOL during cancer survivors' period [20]. When chronic CIPN symptoms become severe, cancer survivors' QOL is lower, and they have problems with mental condition and physical function. We need further study to explore the long-term efficacy of CR oxycodone on chronic CIPN symptoms and QOL in cancer survivors.

\section{Conclusion}

We demonstrated that the $\mathrm{CRC}$ patients who received $\mathrm{CR}$ oxycodone treatment for cancer-related pain had less serious OIPN during FOLFOX chemotherapy. The patients in the OXY group received significantly more cycles of FOLFOX chemotherapy, and a higher cumulative oxaliplatin dose, and therefore, had relatively longer survival than those in the non-OXY group. This study has some limitations. First, it was an observational and retrospective study. Second, the sample size was small.

In conclusion, early administration of CR oxycodone as supportive and palliative care for cancer-related pain and CIPN might be very important and effective e for continuing proactive cancer treatment, which in turn, could lead to a better QOL and prolong survival in advanced CRC patients.

\section{Funding}

The authors received no financial support for the research, authorship, and/or publication of this article.

\section{References}

1. Goldberg RM, Sargent DJ, Morton RF, Fuchs CS, Ramanathan RK, et al. (2004) A randomized controlled trial of fluorouracil plus leucovorin, irinotecan, and oxaliplatin combinations in patients with previously untreated metastatic colorectal cancer. $J$ Clin Oncol 22: 23-30. [Crossref]

2. André T, Boni C, Mounedji-Boudiaf L, et al. (2004) Oxaliplatin, fluorouracil, and leucovorin as adjuvant treatment for colon cancer. $N$ Engl J Med 350: 2343-2351. [Crossref]

3. McWhinney SR, Goldberg RM, McLeod HL (2009) Platinum neurotoxicity pharmacogenetics. Mol Cancer Ther 8: 10-16. [Crossref]

4. Quasthoff S, Hartung HP (2002) Chemotherapy-induced peripheral neuropathy. $J$ Neurol 249: 9-17. [Crossref]

5. Park SB, Goldstein D, Krishnan AV, Lin CS, Friedlander ML, et al. (2013) Chemotherapy-induced peripheral neurotoxicity: a critical analysis. CA Cancer J Clin 63: 419-437. [Crossref]

6. Saif MW, Reardon J (2005) Management of oxaliplatin-induced peripheral neuropathy. Ther Clin Risk Manag 1: 249-258. [Crossref] 
Nagashima M (2018) Survival difference associated with controlled-release oxycodone analgesic therapy for oxaliplatin-induced peripheral neuropathy in advanced colorectal cancer patients

7. Mols F, Beijers T, Lemmens V, van den Hurk CJ, Vreugdenhil G, et al. (2013) Chemotherapy-induced neuropathy and its association with quality of life among 2to 11-year colorectal cancer survivors: results from the population-based PROFILES registry. J Clin Oncol 31: 2699-2707. [Crossref]

8. Tofthagen C, Donovan KA, Morgan MA, Shibata D, Yeh Y (2013) Oxaliplatin-induced peripheral neuropathy's effects on health-related quality of life of colorectal cancer survivors. Support Care Cancer 21: 3307-3313. [Crossref]

9. Hershman DL, Lacchetti C, Dworkin RH, Lavoie Smith EM, Bleeker J, et al. (2014) American Society of Clinical Oncology. Prevention and management of chemotherapyinduced peripheral neuropathy in survivors of adult cancers: American Society of Clinical Oncology clinical practice guideline. J Clin Oncol 32: 1941-1967. [Crossref]

10. Nagashima M, Ooshiro M, Moriyama A, Sugishita Y, Kadoya K, et al. (2014) Efficacy and tolerability of controlled-release oxycodone for oxaliplatin-induced peripheral neuropathy and the extension of FOLFOX therapy in advanced colorectal cancer patients. Support Care Cancer 22: 1579-1584. [Crossref]

11. Gimbel JS, Richards P, Portenoy RK (2003) Controlled-release oxycodone for pain in diabetic neuropathy: a randomized controlled trial. Neurology 60: 927-934. [Crossref]

12. Watson CP, Moulin D, Watt-Watson J, Gordon A, Eisenhoffer J (2003) Controlledrelease oxycodone relieves neuropathic pain: a randomized controlled trial in painful diabetic neuropathy. Pain 105: 71-78. [Crossref]

13. Dubinsky RM, Kabbani H, El-Chami Z, Boutwell C, Ali H, Quality Standards Subcommittee of the American Academy of Neurology (2004) Practice parameter: treatment of postherpetic neuralgia: an evidence-based report of the Quality Standards Subcommittee of the American Academy of Neurology. Neurology. 63: 959-965. [Crossref]
14. Fan BF, OxyContin Tablets Postmarketing Surveillance Study Group China (2008) Postmarketing surveillance study of OxyContin tablets for relieving moderate to severe postherpetic neuralgia pain. Oncology 74: 66-71. [Crossref]

15. Narita M, Nakamura A, Ozaki M, Imai S, Miyoshi K, et al. (2008) Comparative pharmacological profiles of morphine and oxycodone under a neuropathic pain-like state in mice: evidence for less sensitivity to morphine. Neuropsychopharmacology 33 : 1097-1112. [Crossref]

16. Pachman DR, Watson JC, Lustberg MB, Wagner-Johnston ND, Chan A, et al. (2014) Management options for established chemotherapy-induced peripheral neuropathy. Support Care Cancer 22: 2281-2295. [Crossref]

17. Cartoni C, Brunetti GA, Federico V, Efficace F, Grammatico S, et al. (2012) Controlledrelease oxycodone for the treatment of bortezomib-induced neuropathic pain in patients with multiple myeloma. Support Care Cancer 20: 2621-2626. [Crossref]

18. Temel JS, Greer JA, Muzikansky A, Gallagher ER, Admane S, et al. (2010) Early palliative care for patients with metastatic non-small-cell lung cancer. $N$ Engl $J$ Med 363: 733-742. [Crossref]

19. Maione P, Perrone F, Gallo C, Manzione L, Piantedosi F, et al. (2005) Pretreatment quality of life and functional status assessment significantly predict survival of elderly patients with advanced non-small-cell lung cancer receiving chemotherapy: prognostic analysis of the Multicenter Italian Lung Cancer in the Elderly Study. J Clin Oncol 23: 6865-6872. [Crossref]

20. Seretny M, Currie GL, Sena ES, Ramnarine S, Grant R, et al. (2014) Incidence, prevalence, and predictors of chemotherapy-induced peripheral neuropathy: a systematic review and meta-analysis. Pain 155: 2461-2470. [Crossref]

Copyright: $\subseteq 2018$ Nagashima M. This is an open-access article distributed under the terms of the Creative Commons Attribution License, which permits unrestricted use, distribution, and reproduction in any medium, provided the original author and source are credited. 